This document is the accepted manuscript version of a published work that appeared in final form in Nano letters, copyright ( ) American Chemical Society after peer review and technical editing by the publisher.

To access the final edited and published work see: https://dx.doi.org/10.1021/acs.nanolett.5b00708

Published under a "All rights reserved" license. 


\title{
, Phonon Engineering in Isotopically Disordered Silicon Nanowires
}

\author{
2 S. Mukherjee, ${ }^{\dagger}$ U. Givan, ${ }^{\ddagger}$ S. Senz, ${ }^{\ddagger}$ A. Bergeron, ${ }^{\dagger}$ S. Francoeur, ${ }^{\dagger}$ M. de la Mata, ${ }^{\S}$ J. Arbiol, ${ }^{\S}, \|$ \\ ${ }_{3}$ T. Sekiguchi, ${ }^{\perp}$ K. M. Itoh, ${ }^{\perp}$ D. Isheim, ${ }^{\#}$ D. N. Seidman, ${ }^{\#}$ and O. Moutanabbir*, ${ }^{\dagger}$ \\ $4{ }^{\dagger}$ Department of Engineering Physics, Polytechnique Montréal, C. P. 6079, Succ. Centre-Ville, Montréal, Québec H3C 3A7, Canada \\ $5{ }^{\ddagger}$ Max Planck Institute of Microstructure Physics, Weinberg 2, D 06120 Halle (Saale), Germany \\ $6{ }^{\S}$ Institut de Ciencia de Materials de Barcelona, ICMAB-CSIC, Campus de la UAB, 08193 Bellaterra, Catalonia Spain \\ 7 "Institució Catalana de Recerca i Estudis Avançats (ICREA), 08010 Barcelona, Catalonia Spain \\ $8{ }^{\perp}$ Department of Applied Physics and Physico-Informatics, Keio University, Hiyoshi, Yokohama, Japan \\ 9 "Department of Materials Science and Engineering Northwestern University Center for Atom-Probe Tomography, Northwestern \\ 10 University, Evanston, Illinois 60208-3108, United States
}

11 S Supporting Information

12 ABSTRACT: The introduction of stable isotopes in the

13 fabrication of semiconductor nanowires provides an additional

14 degree of freedom to manipulate their basic properties, design

15 an entirely new class of devices, and highlight subtle but

16 important nanoscale and quantum phenomena. With this

17 perspective, we report on phonon engineering in metal-

18 catalyzed silicon nanowires with tailor-made isotopic compo-

19 sitions grown using isotopically enriched silane precursors

$20{ }^{28} \mathrm{SiH}_{4},{ }^{29} \mathrm{SiH}_{4}$, and ${ }^{30} \mathrm{SiH}_{4}$ with purity better than $99.9 \%$. More

21 specifically, isotopically mixed nanowires ${ }^{28} \mathrm{Si}_{x}{ }^{30} \mathrm{Si}_{1-x}$ with a

22 composition close to the highest mass disorder $(x \sim 0.5)$ were

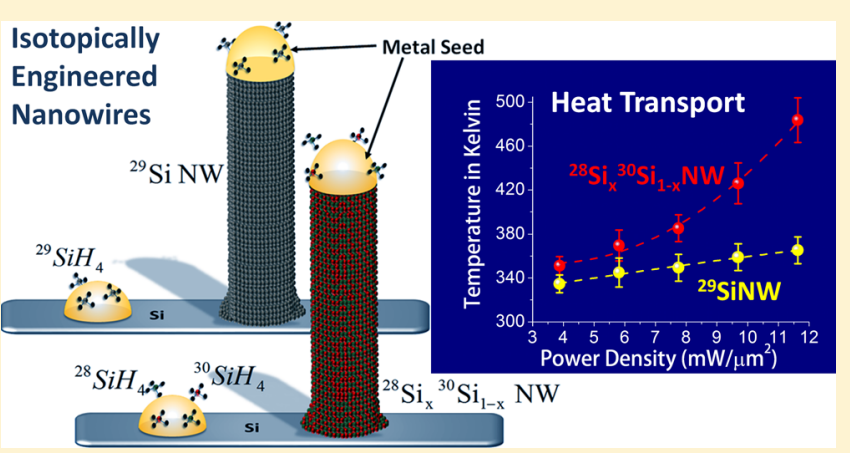
investigated. The effect of mass disorder on the phonon behavior was elucidated and compared to that in isotopically pure ${ }^{29} \mathrm{Si}$ nanowires having a similar reduced mass. We found that the disorder-induced enhancement in phonon scattering in isotopically mixed nanowires is unexpectedly much more significant than in bulk crystals of close isotopic compositions. This effect is explained by a nonuniform distribution of ${ }^{28} \mathrm{Si}$ and ${ }^{30} \mathrm{Si}$ isotopes in the grown isotopically mixed nanowires with local compositions ranging from $x=\sim 0.25$ to 0.70 . Moreover, we also observed that upon heating phonons in ${ }^{28} \mathrm{Si}_{x}^{30} \mathrm{Si}_{1-x}$ nanowires behave remarkably differently from those in ${ }^{29} \mathrm{Si}$ nanowires suggesting a reduced thermal conductivity induced by mass disorder. Using Raman nanothermometry, we found that the thermal conductivity of isotopically mixed ${ }^{28} \mathrm{Si}_{x}{ }^{30} \mathrm{Si}_{1-x}$ nanowires is $\sim 30 \%$ lower than that of isotopically pure ${ }^{29} \mathrm{Si}$ nanowires in agreement with theoretical predictions.

KEYWORDS: Nanowires, stable isotopes, phonons, thermal conductivity, Raman spectroscopy, atom probe tomography

33 Totope engineering in semiconductors, which refers to 34 controlling the content of each stable isotope within a 35 lattice, has been a powerful paradigm to investigate and 36 manipulate some of the important physical properties of 37 semiconductors and exploit them in innovative device 38 structures. $^{1-13}$ Isotopes of an element differ in the number of 39 neutrons in the nucleus. This creates differences between the 40 isotopes in their lattice dynamics and nuclear properties. For 41 instance, the slight difference in zero point motion leads to a 42 difference in atomic volume between the isotope atoms, which 43 influences the lattice constant. ${ }^{3}$ Also, the difference in 44 electron-phonon coupling between crystals of different 45 isotopic composition was found to affect the electronic band 46 gap. ${ }^{4}$ The nuclear spin is another significant difference between 47 stable isotopes. For instance, natural silicon $(\mathrm{Si})$ has three 48 stable isotopes: ${ }^{28} \mathrm{Si},{ }^{29} \mathrm{Si}$, and ${ }^{30} \mathrm{Si}$ with isotopic abundances of 49 92.23, 4.67, and 3.10\%, respectively. Among these three isotopes, only ${ }^{29} \mathrm{Si}$ has a nuclear spin of $1 / 2$, whereas ${ }^{28} \mathrm{Si}$ and 50 ${ }^{30} \mathrm{Si}$ are nuclear spin-free. This property has been crucial in the 51 realization of Si-based quantum information devices. ${ }^{5-8}$ One of 52 the most drastic isotope related effect in semiconductors is 53 found in phonon properties. ${ }^{9-13}$ Mass fluctuation induced by 54 isotope disorder acts as a substitutional defect in a crystal thus 55 affecting the phonon mean free path and consequently the 56 phononic thermal conductivity. Measurements on isotopically 57 pure $\mathrm{Ge}^{9}$ and $\mathrm{Si}^{10}$ crystals showed an enhanced thermal ${ }_{58}$ conductivity as compared to their natural counterparts. Also, 59 lower thermal conductivity was recently demonstrated in $\mathrm{Si} 60$ isotope superlattices. ${ }^{11}$

Received: February 20, 2015

Revised: $\quad$ May 3, 2015 
a)

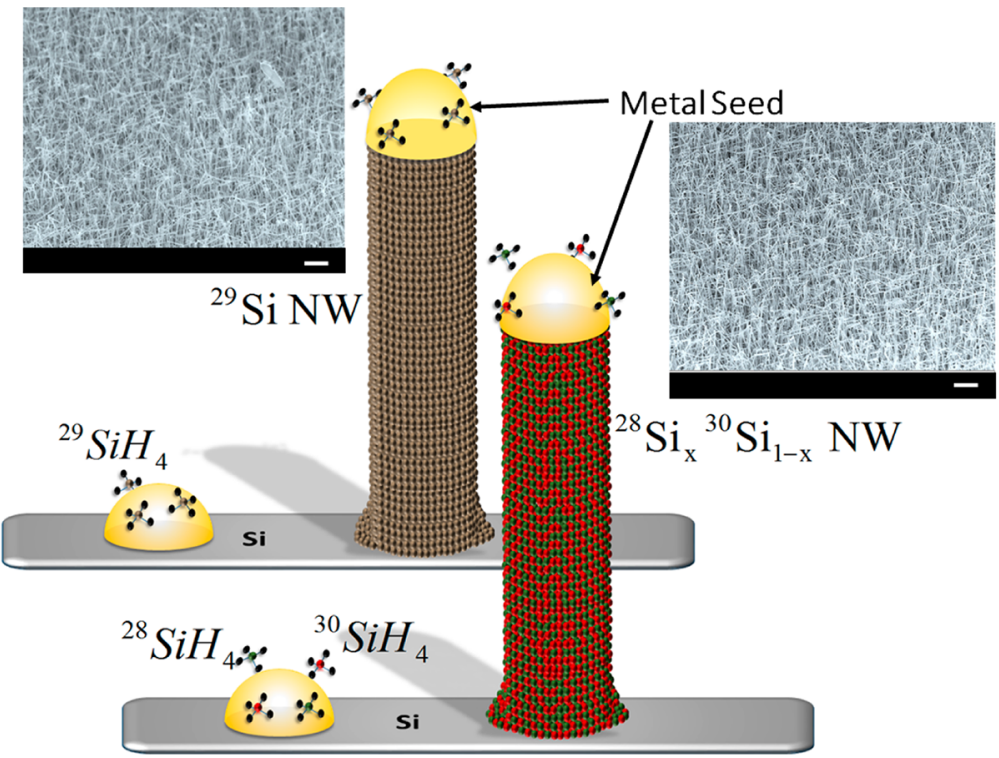

b)

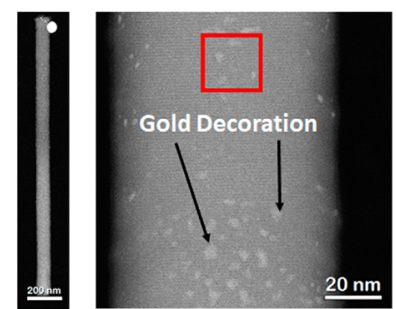

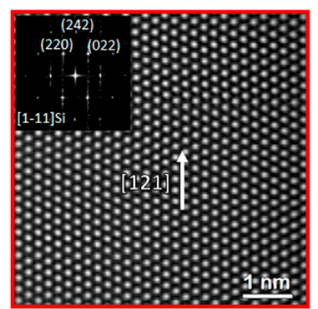

c)

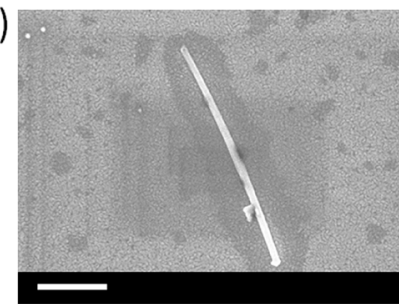

Figure 1. (a) A schematic illustration of the VLS growth of the isotopically engineered Si NWs. Vapor phase precursors are supplied to Au-Si eutectic droplet. For the isotopically pure NWs the precursor is ${ }^{29} \mathrm{SiH}_{4}$ (purity 99.9\%) and for the isotopically mixed $\mathrm{NWs}$, a mixture of ${ }^{28} \mathrm{SiH}_{4}$ (purity 99.99\%) and ${ }^{30} \mathrm{SiH}_{4}$ (purity 99.9\%) was injected. Crystallization of Si atoms from the supersaturated droplet takes place at the dropletnanowire interface which becomes the growth front. Inset: Low-magnification SEM images of as grown isotopically mixed ${ }^{28} \mathrm{Si}_{x}{ }^{30} \mathrm{Si}_{1-x} \mathrm{NWs}$ and isotopically pure ${ }^{29} \mathrm{Si}$ NWs, both recorded at a tilt angle of $60^{\circ}$. The scale bars in both the figures denote $1 \mu \mathrm{m}$. (b) STEM images of the isotopically pure ${ }^{29} \mathrm{Si}$ NWs. Left: A single ${ }^{29} \mathrm{Si}$ NW. The NW have grown along the [121] direction and the image taken from the [11ㅣ] Si zone axis. The scale bar in the figure is $200 \mathrm{~nm}$. Middle: STEM image of the NW sidewalls showing gold decoration on the facets. The scale bar in the figure is $20 \mathrm{~nm}$. Right: High-magnification STEM image (taken from the region marked by the red box in the middle image) and the power spectrum (FFT) in the inset shows the high crystalline quality of the NW. The scale bar in the figure corresponds to $1 \mathrm{~nm}$. (c) SEM image of a single ${ }^{29} \mathrm{Si} \mathrm{NW}$ after sonication and dispersion atop Au capped Si substrate. The scale bar denotes $1 \mu \mathrm{m}$.

62 All the aforementioned properties of semiconductor stable 63 isotopes have been investigated and exploited in bulk materials 64 or thin films. Indeed, conspicuously missing are experimental 65 investigations of the influence of stable isotope impurities on 66 the basic characteristics of nanoscale materials despite the 67 crucial information they could provide concerning their 68 physical properties. Interestingly, there have been only a few 69 theoretical studies on the influence of the isotopic content on 70 basic phonon-related properties of $\mathrm{Si}$ nanowires (NWs). ${ }^{14,15}$ 71 For instance, molecular dynamics (MD) simulations suggested 72 that the thermal conductivity of $\mathrm{Si}$ NWs is reduced 73 exponentially by isotopic impurities at room temperature. ${ }^{14,15}$ 74 In the $\mathrm{MD}$ research, the simulated thermal conductivity of a ${ }_{75}{ }^{28} \mathrm{Si}_{0.5}{ }^{29} \mathrm{Si}_{0.5} \mathrm{NW}$ yields $\sim 80 \%$ of that of isotopically pure ${ }^{28} \mathrm{Si}$ $76 \mathrm{NW}$. Also for a ${ }^{28} \mathrm{Si} /{ }^{29} \mathrm{Si}$ multilayer $\mathrm{NW}$ with a $1.09 \mathrm{~nm}$ period, 77 the calculated thermal conductivity was found to be $\sim 70 \%$ of 78 that of isotopically pure ${ }^{28} \mathrm{Si} \mathrm{NW}^{14}$ Other calculations 79 demonstrate an improvement of more than $25 \%$ in thermo80 electric figure of merit of ${ }^{28} \mathrm{Si}_{0.5}{ }^{29} \mathrm{Si}_{0.5} \mathrm{NWs}$ as compared to a ${ }_{81}{ }^{28} \mathrm{SiNWs} .{ }^{15}$ No experiments have, however, been conducted to 82 elucidate these effects. With this perspective, we report in this 83 work the first experimental investigation of the influence of 84 isotope disorder on the phonon behavior in isotopically 85 engineered Si NWs.
The growth of NWs was carried out using the classical gold- 86 $\mathrm{Au}$ ) catalyzed vapor phase epitaxy using monoisotopic silane 87 ${ }^{28} \mathrm{SiH}_{4},{ }^{29} \mathrm{SiH}_{4}$, and ${ }^{30} \mathrm{SiH}_{4}$ with isotopic purity higher than 88 99.9\%. These precursors were synthesized through the 89 hydrogenation of isotopically enriched $\mathrm{SiF}_{4}{ }^{16}$ The growth 90 conditions are provided in the Supporting Information. Figure $91 \mathrm{f} 1$ 1a illustrates the schematics of the two sets of nanowires $92 \mathrm{f} 1$ investigated in this work. The samples consist of isotopically 93 pure ${ }^{29} \mathrm{Si}$ NWs and isotopically mixed ${ }^{28} \mathrm{Si}_{x}{ }^{30} \mathrm{Si}_{1-x}$ NWs. The 94 former were grown by injecting the monoisotopic ${ }^{29} \mathrm{SiH}_{4} 95$ precursor, whereas for the latter ${ }^{28} \mathrm{SiH}_{4}$ and ${ }^{30} \mathrm{SiH}_{4}$ were 96 simultaneously introduced in the growth chamber. The control 97 of the content of each isotope in the growing isotopically mixed 98 nanowires was achieved through the control of the partial 99 pressures of the two precursors. The low-magnification 100 scanning electron microscope (SEM) images (taken at a tilt 101 of $60^{\circ}$ ) of the ${ }^{28} \mathrm{Si}_{x}{ }^{30} \mathrm{Si}_{1-x}$ NWs and ${ }^{29} \mathrm{Si}$ NWs are displayed in 102 the inset of Figure 1a. The grown NWs are typically $5 \mu \mathrm{m}$ long 103 with a diameter in the $30-100 \mathrm{~nm}$ range. Figure $1 \mathrm{~b}$ shows the 104 scanning transmission electron microscope (STEM) image of a 105 ${ }^{29} \mathrm{Si} \mathrm{NW}$. The NW has grown in the [121] direction and the 106 image is taken from [1시 $\mathrm{Si}$ zone axis. The SEM analysis 107 indicates that while the majority of ${ }^{29} \mathrm{Si}$ NWs have grown along 108 the [111] direction a few have actually grown at an angle of 109 
$110 \sim 19.5^{\circ}$ with respect to the [111] direction corresponding to 111 the [121] crystallographic direction. It is noticeable that the 112 NW surface is decorated with Au clusters mainly near the tip of 113 the NW. This is attributed to Au diffusion from the catalyst 114 droplet along the NW sidewalls during the quenching to room 115 temperature after growth interuption. ${ }^{17,18}$ The high-magnifica116 tion STEM image of the NW included in Figure $1 \mathrm{~b}$ and the 117 power spectrum (fast Fourier transform (FFT)) in the inset 118 show that the grown NWs are of the highest crystalline quality. 119 The STEM analysis of the ${ }^{28} \mathrm{Si}_{x}{ }^{30} \mathrm{Si}_{1-x}$ NWs (not shown here) 120 confirms that the two sets of NWs have identical structural and 121 morphological properties.

122 Raman spectroscopy was employed to investigate the 123 vibrational properties of these NWs. To enable the analysis 124 of individual NWs, the as-grown NWs were first transferred 125 onto Au-capped Si to suppress the background signal from the 126 underlying substrate during subsequent Raman analysis. 127 Backscattering micro-Raman experiments were carried using 128 two laser lines $488 \mathrm{~nm}$ (low power measurements) and $514 \mathrm{~nm}$ 129 (high power measurements) at incident power densities in the 130 range of $0.08-17.76 \mathrm{~mW} / \mu \mathrm{m}^{2}$ (see Supporting Information for 131 more details). The average length of the NWs after dispersion 132 on $\mathrm{Au}$ was found to be on the order of $2-3 \mu \mathrm{m}$. All Raman 133 measurements were performed on single NWs that are in an 134 excellent thermal contact with Au layer (Figure 1c). Clustered 135 and suspended NWs were avoided in this analysis as they heat 136 up faster when exposed to laser beam, which influences their 137 Raman modes. ${ }^{19}$ Figure 2 shows the $\mathrm{Si}-\mathrm{Si}$ LO phonon spectra

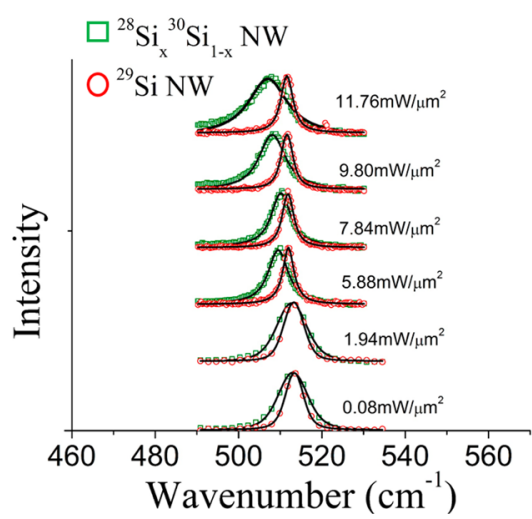

Figure 2. Si-Si LO normalized phonon spectra of ${ }^{28} \mathrm{Si}_{x}{ }^{30} \mathrm{Si}_{1-x} \mathrm{NW}$ and ${ }^{29} \mathrm{Si} \mathrm{NW}$ at different incident laser power densities. The spectra at 1.94 and $0.08 \mathrm{mw} / \mu \mathrm{m}^{2}$ were recorded using the $488 \mathrm{~nm}$ laser and the remaining spectra using the $532 \mathrm{~nm}$ laser. The spectra shown here are representative of a single ${ }^{28} \mathrm{Si}_{x}{ }^{30} \mathrm{Si}_{1-x} \mathrm{NW}$ and a single ${ }^{29} \mathrm{Si} \mathrm{NW}$. The data points for the ${ }^{28} \mathrm{Si}_{x}{ }^{30} \mathrm{Si}_{1-x} \mathrm{NWs}$ are shown as empty green squares and that of ${ }^{29} \mathrm{Si}$ NWs correspond to empty red circles. The black continuous curve corresponds to the Voigt fit.

138 of single ${ }^{28} \mathrm{Si}_{x}{ }^{30} \mathrm{Si}_{1-x}$ and ${ }^{29} \mathrm{Si}$ NWs at different incident laser 139 power densities. A detailed comparison of Raman spectra of ${ }_{140}{ }^{28} \mathrm{Si}_{x}{ }^{30} \mathrm{Si}_{1-x}$ and ${ }^{29} \mathrm{Si}$ single $\mathrm{NWs}$ at low power regime is 141 provided in Figure S1 (Supporting Information). Note that all 142 effects related to phonon confinement are excluded here as the 143 diameter of the investigated NWs is larger than the phonon 144 mean free path in $\mathrm{Si}$ around room temperature. Recorded 145 spectra were fitted with Voigt line profiles to extract peak 146 positions and full width at half-maximum (fwhm).

147 A first analysis of the data is based on the quasi-harmonic 148 approximation, which is a valid approximation for semi- conductors at room temperature. ${ }^{20}$ Herein, it is important to 149 minimize the excess local heating of the NWs, which would 150 occur when the incident laser power is sufficiently high. Hence, 151 all calculations involving the quasi-harmonic approximation 152 were carried out on data sets recorded at the lowest incident 153 power density of $0.08 \mathrm{~mW} / \mu \mathrm{m}^{2}$ at which the local temperature 154 of the NWs is confirmed to be equal to the ambient 155 temperature of $300 \mathrm{~K}$ (Supporting Information). A close 156 inspection of the spectra exhibited in Figure 2 shows two 157 separate phonon related effects. First, at all incident laser 158 powers the Raman spectrum for ${ }^{28} \mathrm{Si}_{x}{ }^{30} \mathrm{Si}_{1-x} \mathrm{NWs}$ is always 159 broader and red-shifted as compared to the spectrum of ${ }^{29} \mathrm{Si} 160$ NWs. Second, regardless of the type of the NW, as the incident 161 power increases, all peaks broaden and redshift. This effect is 162 due to laser-induced heating of the NWs. Figure 3 depicts the $163 \mathrm{f} 3$ evolution of the average peak position and the evolution of the 164 average fwhm with incident power density for both ${ }^{28} \mathrm{Si}_{x}{ }^{30} \mathrm{Si}_{1-x}{ }_{165}$ and ${ }^{29} \mathrm{Si}$ NWs. In Figure 3a,b are displayed the data recorded at 166 low laser power densities averaged over a large number ( $>10$ of 167 single NWs). High-power measurements are given in Figure 168 3d,e. The peak position and fwhm of $4-5$ individual ${ }^{28} \mathrm{Si}_{x}{ }^{30} \mathrm{Si}_{1-x} 169$ NWs and ${ }^{29} \mathrm{Si}$ NWs, as extracted from the Voigt fit of the raw 170 data, at both low and high power levels are displayed in Figure 171 S2 (Supporting Information). Interestingly, both the broad- 172 ening and the redshift are found to be more pronounced for 173 ${ }^{28} \mathrm{Si}_{x}{ }^{30} \mathrm{Si}_{1-x}$ NWs. For instance, the average peak position within 174 the laser power range investigated varies by about $4 \mathrm{~cm}^{-1}$ for 175 ${ }^{28} \mathrm{Si}_{x}{ }^{30} \mathrm{Si}_{1-x}$ NWs as compared to $\sim 1 \mathrm{~cm}^{-1}$ for ${ }^{29} \mathrm{Si} \mathrm{NWs}$. This 176 indicates that the two types of NWs react differently to local 177 heating induced by laser.

In the following, we elucidate the origin of the remarkable 179 changes in Raman spectra as a function of the NW isotopic 180 content. According to the virtual crystal approximations 181 (VCA), a simple harmonic analysis predicts that the energy 182 of a phonon mode is inversely proportional to the square root 183 of the average isotopic mass ${ }^{1}-\omega_{\text {phonon }} \propto(1 /\langle m\rangle)^{1 / 2}$. Here $m$ is 184 the average isotopic mass given by $\langle m\rangle=\sum_{i} c_{i} m_{i}$, with $c_{i}$ being 185 the fractional composition of an isotope of mass $m_{i}$. Using the 186 ratio of the average peak position at the lowest incident power 187 density of $0.08 \mathrm{~mW} / \mu \mathrm{m}^{2}$ (Figure $3 \mathrm{a}$ ) and the value of $\langle m\rangle_{29-\mathrm{Si}}, 188$ we computed $\langle m\rangle_{\text {Iso-mix }}=29.05 \mathrm{amu}$. Thus, the corresponding 189 fractional composition of ${ }^{28} \mathrm{Si}$ in the isotopically mixed NWs is 190 $x=0.47 \pm 0.07$ calculated from the known values of $\langle m\rangle_{28-S i} 191$ and $\langle m\rangle_{30-S \mathrm{i}}$ in the identity: $\langle m\rangle_{\text {Iso-mix }}=x \times\langle m\rangle_{28-S \mathrm{Si}}+(1-x) 192$ $\times\langle m\rangle_{30-S i}$. Note that composition calculated employing the 193 quasi-harmonic approximation is always an average estimate. 194

In Figure 3a, the shift rate of the average peak position with 195 power density up to $5-6 \mathrm{~mW} / \mu \mathrm{m}^{2}$ is only slightly higher for 196 ${ }^{28} \mathrm{Si}_{x}{ }^{30} \mathrm{Si}_{1-x}$ NWs as compared to ${ }^{29} \mathrm{Si} \mathrm{NWs}$ (the corresponding ${ }_{197}$ slopes of the dotted lines are 0.38 and $0.30\left(\mathrm{~cm}^{-1} \mu \mathrm{m}^{2}\right) /(\mathrm{mW}), 198$ respectively). At higher power densities, the behavior of 199 ${ }^{28} \mathrm{Si}_{x}{ }^{30} \mathrm{Si}_{1-x} \mathrm{NWs}$ is markedly different from ${ }^{29} \mathrm{Si} \mathrm{NWs}$ with 200 the average phonon frequency of the former undergoing a 201 drastic redshift as compared to the latter (Figure 3d). The 202 evolution of fwhm follows the same trend as the shift in 203 phonon frequency. Below $\sim 6 \mathrm{~mW} / \mu \mathrm{m}^{2}$, the average fwhm of 204 ${ }^{28} \mathrm{Si}_{x}{ }^{30} \mathrm{Si}_{1-x}$ NWs and ${ }^{29} \mathrm{Si}$ NWs evolve qualitatively similarly 205 with incident power density. At all incident power densities the 206 spectra of ${ }^{28} \mathrm{Si}_{x}{ }^{30} \mathrm{Si}_{1-x}$ NWs are always broader than that of ${ }^{29} \mathrm{Si} 207$ NWs. This can be also verified from the respective spectra 208 displayed in Figure 2. At low and mid power ranges, the average 209 fwhm of the former is about $3-3.5 \mathrm{~cm}^{-1}$ broader than the 210 
a)

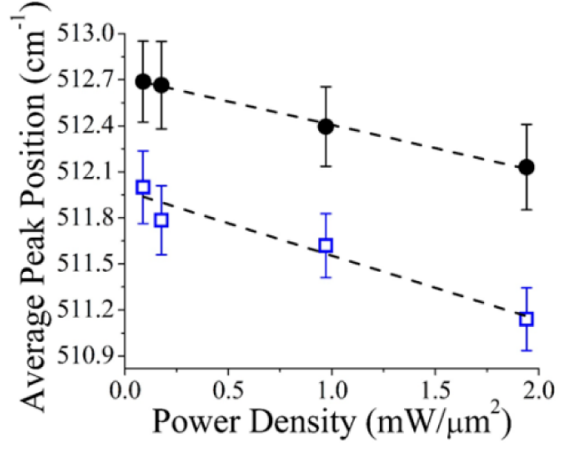

b)

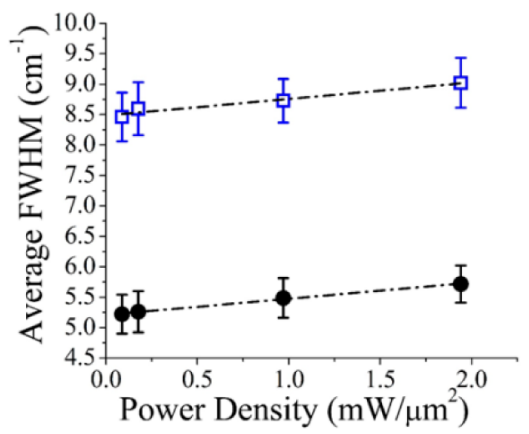

c)

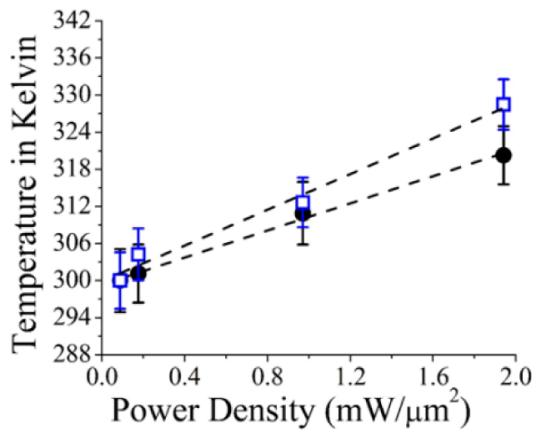

d)

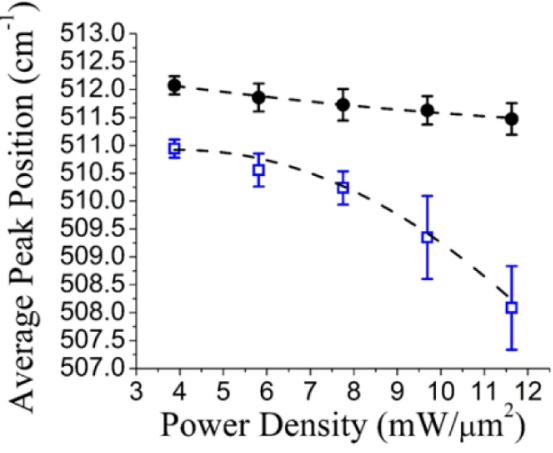

e)

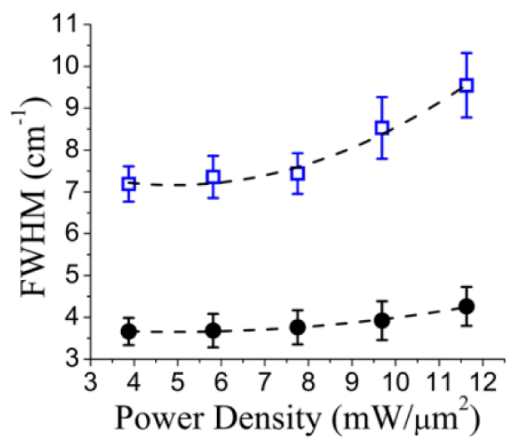

f)

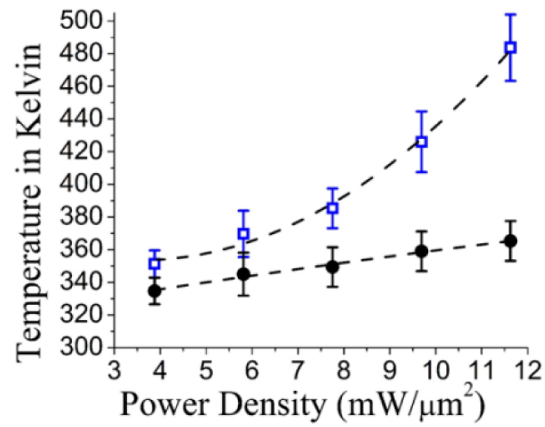

Figure 3. (a,b) Measurements using $488 \mathrm{~nm}$ laser at low incident power density; (d,e) measurements using $532 \mathrm{~nm}$ laser at high incident power density. In all figures, the empty blue squares correspond to the isotopically mixed ${ }^{28} \mathrm{Si}_{x}{ }^{30} \mathrm{Si}_{1-x} \mathrm{NWs}$ and the filled black circles represent the isotopically pure ${ }^{29} \mathrm{Si}$ NWs. (a,d) Evolution of average peak position with incident laser power density for both ${ }^{28} \mathrm{Si}_{x}{ }^{30} \mathrm{Si}_{1-x} \mathrm{NWs}$ and ${ }^{29} \mathrm{Si}_{\mathrm{NW}} \mathrm{NW}$. (b,e) Evolution of the average fwhm with incident laser power density for both ${ }^{28} \mathrm{Si}_{x}{ }^{30} \mathrm{Si}_{1-x} \mathrm{NWs}$ and ${ }^{29} \mathrm{Si} \mathrm{NWs}$. In panels a and $\mathrm{b}$, the averaging was done over measurements on more than 10 single NWs, and in panels $\mathrm{d}$ and e the averaging was done over measurements on 7 single NWs. The error bars in panels $a, b, d$, and e are double the standard deviation of the peak position and fwhm from respective average values. (c,f) Plots of the effective local temperature of the NWs extracted from the shift in average peak position in panels a and d, respectively. The error bars represent the uncertainty in the calculated temperature due to the standard deviation of the measured peak position. The dotted lines in panels a-f are guides to the eye.

211 latter. A detailed discussion on the origin of this effect will be 212 presented later in the text. As it can be seen in Figure $3 \mathrm{e}$, at 213 high power regime the spectra of ${ }^{28} \mathrm{Si}_{x}{ }^{30} \mathrm{Si}_{1-x}$ NWs start to 214 broaden much rapidly as compared to ${ }^{29} \mathrm{Si}$ NWs. The difference 215 in absolute values of the average fwhm between Figure 3, panels $216 \mathrm{~b}$ and e, comes simply from the difference in spectral resolution 217 between the two setups (Supporting Information).

218 The redshift in peak position and broadening of Raman 219 spectra with increasing power densities are indicative of how 220 the NWs are reacting to laser-induced heating. From Figure 3, 221 it can be seen that the behavior of the two types of NWs only 222 show slight differences at low power, but at high power ${ }_{223}{ }^{28} \mathrm{Si}_{x}{ }^{30} \mathrm{Si}_{1-x}$ NWs are much more affected than ${ }^{29} \mathrm{Si}$ NWs. A 224 convenient way to quantify this heating effect is to extract the $225 \mathrm{NW}$ local temperature. Herein, an estimate of the effective local temperature is made from the shift in average peak position 226 with the incident laser power. The peak position of a NW, 227 $\Omega(T)$ at a temperature " $T$ ", is given by ${ }^{21} \Omega(T)=\omega_{0}+\prod(T), 228$ where $\omega_{0}$ is the peak position at $0 \mathrm{~K}$ and $\prod(T)$ is the shift of 229 peak position at $T$, given by

$$
\begin{aligned}
\Pi(T)= & C\left[1+\frac{2}{\mathrm{e}^{\hbar \omega_{0} / 2 k_{\mathrm{B}} T}-1}\right] \\
& +D\left[1+\frac{3}{\mathrm{e}^{\hbar \omega_{0} / 3 k_{\mathrm{B}} T}-1}+\frac{3}{\left(\mathrm{e}^{\hbar \omega_{0} / 2 k_{\mathrm{B}} T}-1\right)^{2}}\right]
\end{aligned}
$$

where " $C$ " and " $D$ " are constants. The first term is related to 232 three-phonon anharmonic interaction and the second term 233 represents the four phonon interaction. The probability of the 234 latter being small, we can reasonably neglect it to be left with 235 the first term in the right-hand side of (1). Balkanski et al. 236 
237 calculated the phonon frequency for ${ }^{\mathrm{Nat}} \mathrm{Si}$ at $0 \mathrm{~K},{ }^{21} \omega_{0}^{\mathrm{Nat}-\mathrm{Si}}=$ $238529 \mathrm{~cm}^{-1}$ (using only the three phonon process). By taking into 239 account the change in the reduced mass, we calculate $\omega_{0}^{\text {Iso-mix }}$ 240 (for ${ }^{28} \mathrm{Si}_{x}{ }^{30} \mathrm{Si}_{1-x} \mathrm{NWs}$ ) and $\omega_{0}^{29-\mathrm{Si}}$ (for ${ }^{29} \mathrm{Si} \mathrm{NWs}$ ) to be 519.80 241 and $520.81 \mathrm{~cm}^{-1}$, respectively. $C$ was estimated from the data 242 recorded at the lowest laser power density $\left(0.08 \mathrm{~mW} / \mu \mathrm{m}^{2}\right)$ 243 corresponding to a temperature of $300 \mathrm{~K}$. Next, $\prod(T)$ was 244 calculated from Figure $3 a, b$ for different incident power 245 densities and the NW local temperature was then estimated 246 as displayed in Figure 3c,f. The plot reveals that ${ }^{28} \mathrm{Si}_{x}{ }^{30} \mathrm{Si}_{1-x}$ 247 NWs are getting slightly more heated up as compared to ${ }^{29} \mathrm{Si}$ $248 \mathrm{NWs}$ in the low power density regime $\left(<6 \mathrm{~mW} / \mathrm{cm}^{2}\right)$, the 249 temperature of the former is higher by $\sim 10-15 \mathrm{~K}$ at $1.94 \mathrm{~mW} /$ $250 \mu \mathrm{m}^{2}$ and $\sim 25-30 \mathrm{~K}$ at $5.88 \mathrm{~mW} / \mu \mathrm{m}^{2}$ as compared to the latter. 251 However, at the highest power of $11.76 \mathrm{~mW} / \mu \mathrm{m}^{2}$ the 252 difference in temperatures is quite significant. Indeed, the 253 temperature of ${ }^{28} \mathrm{Si}_{x}{ }^{30} \mathrm{Si}_{1-x}$ NWs is almost $120 \mathrm{~K}$ higher than 254 that of ${ }^{29} \mathrm{Si}$ NWs. At low power regime, the rate of increase of 255 temperature with increasing laser power, $\Delta T / \Delta P$, is $\sim 1.30$ 256 times higher for ${ }^{28} \mathrm{Si}_{x}{ }^{30} \mathrm{Si}_{1-x}$ NWs than ${ }^{29} \mathrm{Si} \mathrm{NWs}$ and becomes $257 \sim 6$ times larger in the high power regime $\left(6-12 \mathrm{~mW} / \mu \mathrm{m}^{2}\right)$.

258 To evaluate the change in thermal conductivity between the 259 two sets of NWs, which led to the result described above, we 260 used Raman nanothermometry ${ }^{22,23}$ in conjunction with a heat 261 transport model. This model draws its basis from the 262 assumptions that the region of the NW exposed to laser acts 263 as the heat source and that the major portion of the generated 264 heat is dissipated by conduction along the NW growth axis and 265 at the NW-Au interface (Supporting Information Figure S3). 266 Details of the model are provided in the Supporting 267 Information. Around $300 \mathrm{~K}$, based on the rate of increase of 268 temperature, we estimated the ratio of thermal conductivities of ${ }_{269}^{29} \mathrm{Si}$ NWs to ${ }^{28} \mathrm{Si}_{x}{ }^{30} \mathrm{Si}_{1-x} \mathrm{NWs} \kappa_{\mathrm{Si}-29} / \kappa_{\text {Iso-Mix }}$ to be $\sim 1.30$. This 270 means there is a $\sim 30 \%$ decrease in $\kappa_{\text {Iso-Mix }}$ as compared to $271 \kappa_{\mathrm{Si}-29}$. Interestingly, this value is close to the theoretical 272 prediction of $30 \%$ reduction in case of ${ }^{28} \mathrm{Si}_{0.5}{ }^{30} \mathrm{Si}_{0.5}$ bulk alloys as 273 compared to ${ }^{\mathrm{Nat}} \mathrm{Si}$ at room temperature, ${ }^{24}$ but it is slightly 274 higher than $\sim 20 \%$ reduction predicted for ${ }^{28} \mathrm{Si}_{0.5}{ }^{29} \mathrm{Si}_{0.5} \mathrm{NW}$ as 275 compared to ${ }^{28} \mathrm{Si} \mathrm{NW}$ at $300 \mathrm{~K}^{14}$ It is also noteworthy that the 276 observed mass disorder-induced change in Si NW thermal 277 conductivity is lower the $50 \%$ reduction demonstrated for ${ }_{278}{ }^{12} \mathrm{C}_{0.5}{ }^{13} \mathrm{C}_{0.5}$ graphene as compared to purified ${ }^{13} \mathrm{C}$ graphene. ${ }^{25}$ 279 For Si NWs, Yang and co-workers predicted that at room 280 temperature a much significant reduction in thermal con281 ductivity up to $\sim 70 \%$ can be achieved when a ${ }^{28} \mathrm{Si} \mathrm{NW}$ is mixed 282 at $50 \%$ with ${ }^{42} \mathrm{Si}$ atoms. ${ }^{14}$ However, ${ }^{42} \mathrm{Si}$ being radioactive with 283 a half-life of $\sim 13 \mathrm{~ms}$ cannot obviously be implemented for any 284 practical purpose. Note that the ratio of thermal conductivity 285 was specifically calculated in the low power regime because the 286 fluctuations in the measured peak position of different ${ }_{287}^{28} \mathrm{Si}_{x}{ }^{30} \mathrm{Si}_{1-x}$ NWs at high power regime are very large 288 (Supporting Information Figure $\mathrm{S} 2: \mathrm{Ab}$ ) thus making the 289 estimation of the temperature of ${ }^{28} \mathrm{Si}_{x}{ }^{30} \mathrm{Si}_{1-x}$ NWs fraught 290 with large uncertainties.

291 Although our experimental data are consistent with early 292 theoretical predictions, isotope mixing alone cannot explain all 293 the observed differences in phonon properties between 294 isotopically disordered and pure NWs. In Figure $3 b$, the $295 \mathrm{fwhm}$ of ${ }^{28} \mathrm{Si}_{x}{ }^{30} \mathrm{Si}_{1-x} \mathrm{NWs}$ is consistently larger by almost 3-3.5 $296 \mathrm{~cm}^{-1}$ at all incident power compared to the ${ }^{29} \mathrm{Si} \mathrm{NWs}$. As 297 phonon confinement is excluded here, broadening of a Raman 298 spectrum occurs due to scattering of phonons. In general, the greater the broadening of a Raman line, the greater is the rate of 299 phonon scattering in the material. At a first glance, it appears 300 that excess broadening is due to the sole effect of scattering of 301 phonons from the mass disorder but our analysis suggests that 302 this contribution alone is not sufficient. Herein, in order to 303 quantify the effect of mass disorder we first compare our data 304 with the results of Raman measurements on isotopically 305 engineered bulk samples. ${ }^{26}$ In those studies, Cardona and co- 306 workers measured bulk $\mathrm{Si}$ samples of different isotopic 307 composition. The Raman spectra of a material has a slight 308 dependence on the excitation wavelength ${ }^{27}$ but at low 309 incidence power this dependence can be neglected. We focus 310 mainly on bulk ${ }^{28} \mathrm{Si}_{0.5}{ }^{30} \mathrm{Si}_{0.5}$ sample as it has almost the same 311 average isotopic composition as our isotopically mixed NWs 312 and hence nearly the same mass variance, $g_{2}$, which is given by 313

$$
g_{2}=\frac{\sum_{i} c_{i} m_{\mathrm{i}}^{2}-\left(\sum_{\mathrm{i}} c_{i} m_{i}\right)^{2}}{\left(\sum_{i} c_{i} m_{i}\right)^{2}}=\frac{\left\langle m^{2}\right\rangle-\left\langle m^{2}\right\rangle}{\left\langle m^{2}\right\rangle}
$$

There are various scattering mechanisms for phonons in a 315 material. The first is the anharmonic scattering of a LO zone- 316 center phonon into two or three phonons with larger wave 317 vector and smaller energy. Anharmonic scattering increases 318 with temperature and at a given temperature the rate of 319 anharmonic scattering of phonons is inversely proportional to 320 the average isotopic mass. ${ }^{26}$ The second is the isotope 321 scattering of phonons that is proportional to $g_{2}{ }^{28}$ The third 322 is the Umklapp scattering of two phonons, which produces a 323 third phonon outside the first Brillouin zone. Umklapp 324 scattering becomes important for temperatures above the 325 Debye temperature, which is $645 \mathrm{~K}$ for $\mathrm{Si}^{29}$ Thus, this 326 scattering is irrelevant in our case as the temperature of the 327 analyzed NWs remains significantly below this temperature 328 (Figure 3). The fourth is the surface scattering of phonons, 329 which is also temperature independent but depends inversely 330 on the size of the material under consideration. ${ }^{30}$ Surface 331 scattering can be neglected for bulk materials but not for NWs. 332 The fifth is the Fano scattering, which is the scattering of 333 phonons from thermally generated electron hole pairs. ${ }^{31} 334$ Because Fano scattering is significant only at high levels of 335 carrier injection, it can be neglected for intrinsic Si NWs 336 investigated in this work. This leaves us with just two scattering 337 mechanisms in bulk samples, the anharmonic scattering and 338 scattering from isotope disorder. We extracted from ref 26 that 339 for the bulk ${ }^{28} \mathrm{Si}_{0.50}{ }^{30} \mathrm{Si}_{0.50}$ sample, $\Delta_{\text {Anhrm }}^{\text {Iss-Mix Bulk }}$, the contribution 340 of anharmonic scattering of phonons and $\Delta_{\text {Isotope }}^{\text {Iso Mulk }}$, the 341 contribution of isotope scattering of phonons to the total line 342 broadening at $T=6 \mathrm{~K}$ are about 1.16 and $0.065 \mathrm{~cm}^{-1}, 343$ respectively. We extrapolated $\Delta_{\text {Anhrm }}^{\text {Iso-Mix Bulk }}$ to $T=300 \mathrm{~K}$, using 344 the following equation ${ }^{21}$

$$
\Delta_{\text {Anhrm }}(T)=A\left[1+\frac{2}{\mathrm{e}^{\hbar \omega_{0} / 2 \mathrm{k}_{\mathrm{B}} \mathrm{T}}-1}\right]
$$

Similar to eq 1 we have neglected the four phonon 347 interaction and normalized the data for $\Delta_{\text {Anhrm }}^{\text {Iso-Mix Bulk }}$ at $T=6348$ $\mathrm{K}$ to find the constant “ $A$ ". $\omega_{0}$ for the bulk ${ }^{28} \mathrm{Si}_{0.50}{ }^{30} \mathrm{Si}_{0.50}$ sample 349 was calculated by the same approach used to calculate $\omega_{0}^{\text {Iso-Mix }} 350$ and $\omega_{0}^{29-S i}$. We found $\Delta_{\text {Anhrm }}^{\text {Iso-Mix Bulk }}(T=300 \mathrm{~K})=1.36 \mathrm{~cm}^{-1} .351$ The difference in spectral resolution between our setup and the 352 setup used in ref 26 is accounted for in this analysis. ${ }^{32,33} 353$ Summing up the discussion in form of equations, the fwhm of 354 bulk ${ }^{28} \mathrm{Si}_{0.50}{ }^{30} \mathrm{Si}_{0.50}$ sample consists of two contributions 
a)
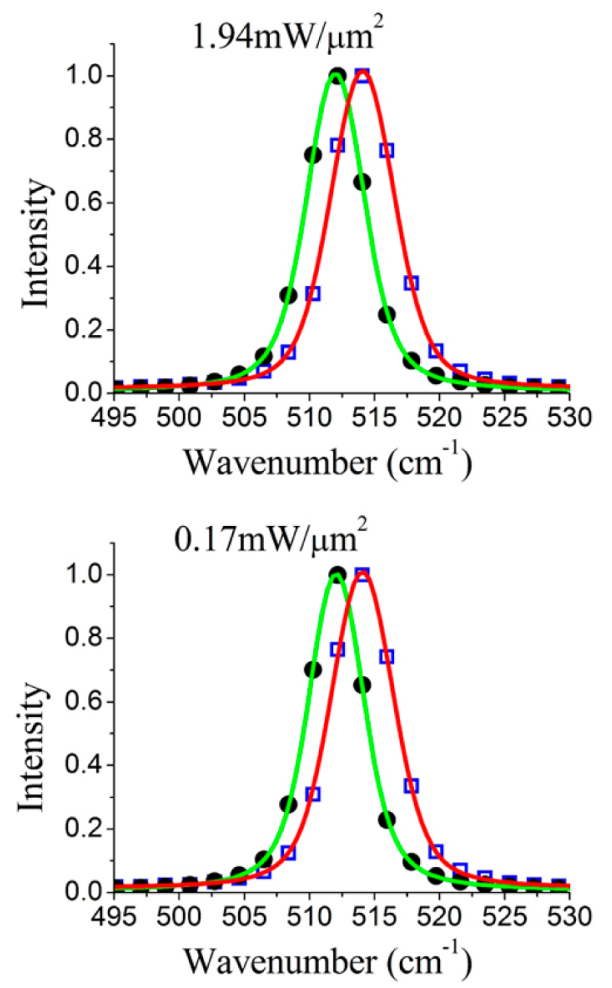

b)

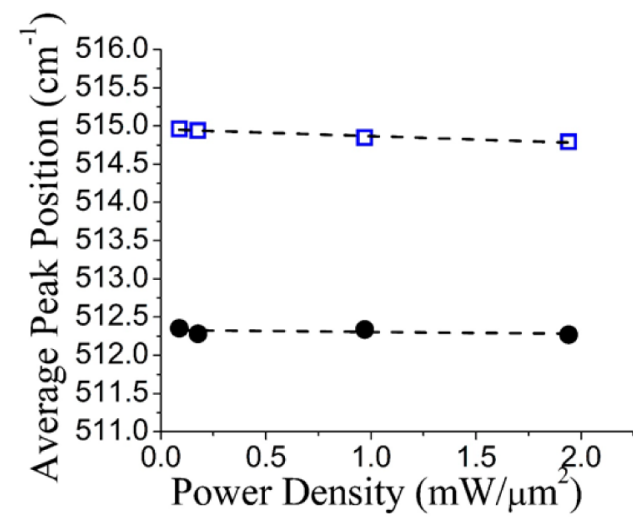

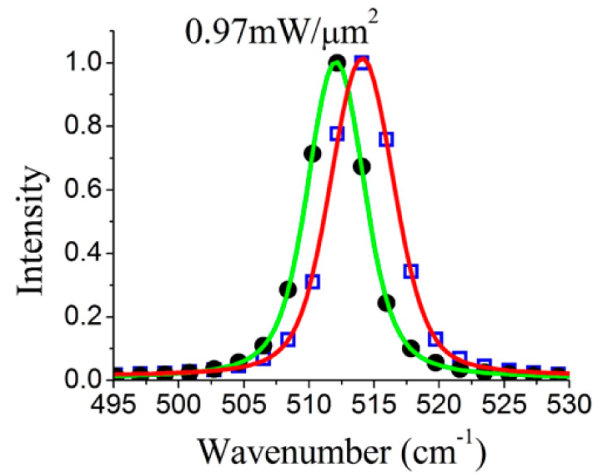

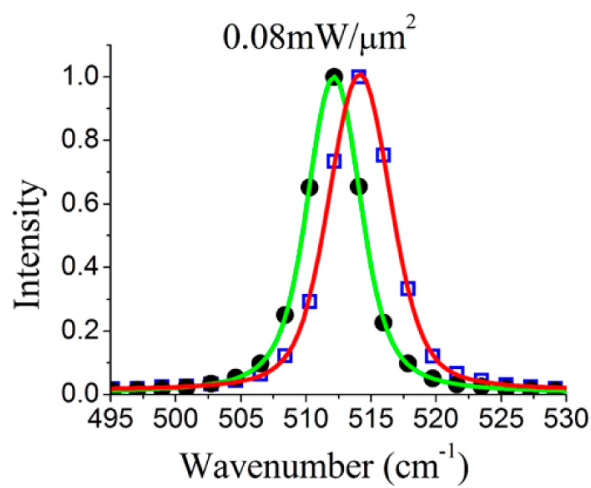

C)

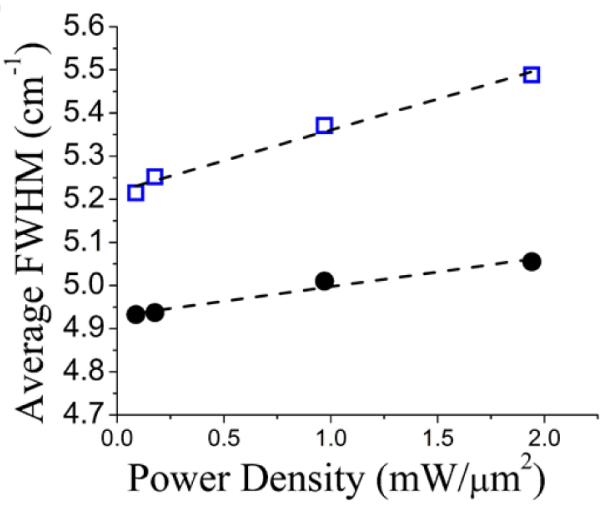

Figure 4. (a) $\mathrm{Si}-\mathrm{Si} \mathrm{LO}$ normalized phonon spectra of ${ }^{28} \mathrm{Si}_{0.6}{ }^{30} \mathrm{Si}_{0.4}$ and ${ }^{29} \mathrm{Si}$ bulk crystals at different incident laser (488 nm) power densities: 1.94 , $0.97,0.17$, and $0.08 \mathrm{~mW} / \mu \mathrm{m}^{2}$. (b) Evolution of average peak position and (c) evolution of average fwhm with incident laser power density for both ${ }^{28} \mathrm{Si}_{0.6}{ }^{30} \mathrm{Si}_{0.4}$ and ${ }^{29} \mathrm{Si}$ bulk samples extracted from the corresponding Voigt fits. In all of the three figures the data points for the ${ }^{28} \mathrm{Si}_{0.6}{ }^{30} \mathrm{Si}_{0.4}$ bulk sample are shown in empty blue squares and that of ${ }^{29} \mathrm{Si}$ bulk sample are shown in filled black circles. The averaging was done over measurements on four different spots on each sample. In (a), the red and the green curves correspond the Voigt fit of the respective raw data. In (b,c), the error bars in both the figures are smaller than the data symbols used. The dotted lines in both the figures are guide to the eye.

$$
\mathrm{fwhm}_{\text {Bulk }}(T=300 \mathrm{~K})=\Delta_{\text {Anhrm }}^{\text {Iso-Mix Bulk }}(T=300 \mathrm{~K})+\Delta_{\text {Isotope }}^{\text {Iso-Mix Bulk }}
$$

357 In comparison, the fwhm of isotopically mixed ${ }^{28} \mathrm{Si}_{x}{ }^{30} \mathrm{Si}_{1-x}$ $358 \mathrm{NWs}$, after correcting for the spectral resolution, consists of 359 three contributions

360

$$
\begin{aligned}
\text { fwhm }_{\text {Iso-Mix NW }}= & \Delta_{\text {Anhrm }}^{\text {Iso-Mix NW }}(T=300 \mathrm{~K}) \\
& +\Delta_{\text {Isotope }}^{\text {Iso-Mix NW }}+\Delta_{\text {Surface }}^{\text {Iso-Mix NW }}
\end{aligned}
$$

$361 \Delta_{\text {Surface }}^{\text {Iso-Mix } N W}$ is the broadening due to surface scattering of 362 phonons. This contribution is peculiar to NWs but absent for 363 bulk materials. Similarly for the fwhm of the isotopically pure $364{ }^{29} \mathrm{Si}$ NWs

$$
\mathrm{fwhm}_{\mathrm{Si}-29 \mathrm{NW}}=\Delta_{\text {Anhrm }}^{\mathrm{Si}-29 \mathrm{NW}}(T=300 \mathrm{~K})+\Delta_{\text {Surface }}^{\mathrm{Si}-29 \mathrm{NW}}
$$

Next, we take $\left.\Delta_{\text {Anhrm }}^{\text {Iso-Mix Bulk }}\right|_{\mathrm{T}}=\left.\Delta_{\text {Anhrm }}^{\text {Iso-Mix NW }}\right|_{\mathrm{T}}$ and $\Delta_{\text {Isotope }}^{\text {Iso-Mix Bulk }} 366$ $=\Delta_{\text {Isotope }}^{\text {Iso-Mix NW }}$, because the anharmonic scattering and isotope 367 scattering of phonons depend on the temperature and isotopic 368 composition, but not on the size of the material as long as 369 confinement effects are unimportant. Thus, $\Delta_{\text {Isotope }}^{\text {Iso-Mix NW }}=0.065370$ $\mathrm{cm}^{-1}$ and at the lowest incident laser power density, 371 corresponding to a temperature of about $300 \mathrm{~K}, \Delta_{\text {Anhrm }}^{\text {Iso-Mix NW }} 372$ is $1.36 \mathrm{~cm}^{-1}$. We can now relate $\Delta_{\text {Anhrm }}^{\mathrm{Si}-29 \mathrm{NW}}$ to $\Delta_{\text {Anhrm }}^{\text {Iso-Mix NW }} 373$ through the inverse mass relation at a fixed temperature 374 $\left.\Delta_{\text {Anhrm }}\right|_{T} \propto 1 /\langle m\rangle$, giving $\Delta_{\text {Anhrm }}^{\text {Si-29 NW }}(T=300 \mathrm{~K}) \times \Delta_{\text {Anhrm }}^{\text {Iso-Mix NW }} 375$ $(T=300 \mathrm{~K}) \times\left(\langle m\rangle_{\text {Iso-Mix }} /\langle m\rangle_{\mathrm{Si}-29}\right) \approx 1.36 \mathrm{~cm}^{-1}$. Putting the 376 value of the fwhm of ${ }^{29} \mathrm{Si}$ NWs at the lowest incident power and 377 $\Delta_{\text {Anhrm }}^{\mathrm{Si}-29 \mathrm{NW}}$ in eq 6 we deduce the contribution of surface 378 scattering to the NWs ${ }^{29} \mathrm{Si}-{ }^{29} \mathrm{Si}$ Raman peak broadening: 379 $\Delta_{\text {Surface }}^{\mathrm{Si}-29 \mathrm{NW}} 0.88 \mathrm{~cm}^{-1}$. Note that the contribution of surface 380 scattering of phonons, which is diameter dependent, is the same 381 
382 for both types of NWs investigated in this work as they have 383 comparable diameters. It is therefore reasonable to conclude 384 that the surface induced broadening of Raman spectra is the 385 same for both types of NWs, that is $\Delta_{\text {Surface }}^{\text {Iso-Mix NW }} \approx \Delta_{\text {Surface }}^{\mathrm{Si}-29 \mathrm{NW}}=$ $3860.88 \mathrm{~cm}^{-1}$. Now, the left-hand side (LHS) of eq 5 at the lowest 387 incident laser power density is $5.5 \mathrm{~cm}^{-1}$, whereas the right-hand 388 side (RHS) after summing up $\Delta_{\text {Anhrm }}^{\text {Iso-Mix NW }}, \Delta_{\text {Isotope }}^{\text {Iso-Mix NW }}$, and $389 \Delta_{\text {Surface }}^{\text {Iso-Mix NW }}$ equates to $2.3 \mathrm{~cm}^{-1}$. The fact that the equality of 390 LHS and RHS in eq 5 does not hold suggests that there must 391 be some other source of spectral broadening that has not been 392 considered in eq 5 . We rule out the possibilities of phonons 393 scattering at crystallographic defects because the two sets of 394 NWs are of high crystalline quality. This suggests that the 395 excess broadening is induced by effects other than those listed 396 above.

397 To verify the calculations above, we performed a series of 398 control experiments on isotopically mixed and isotopically pure 399 bulk samples. These isotopically engineered bulk crystals were 400 grown by floating zone technique. The secondary ion mass 401 spectrometer analysis (not shown here) estimated that the 402 isotopically mixed bulk sample is composed of about $60 \%$ of ${ }_{403}{ }^{28} \mathrm{Si}$ and $40 \%$ of ${ }^{30} \mathrm{Si}$. The spectra of both ${ }^{29} \mathrm{Si}$ and ${ }^{28} \mathrm{Si}_{0.6}{ }^{30} \mathrm{Si}_{0.4}$ 404 bulk samples at four different laser power densities are shown in 405 Figure 4a. For these crystals, the average mass of the ${ }_{406}{ }^{28} \mathrm{Si}_{0.6}{ }^{30} \mathrm{Si}_{0.4}$ sample is slightly smaller than that of ${ }^{29} \mathrm{Si}$ bulk 407 sample. Consequently, the spectra of the former are blueshifted 408 at all incident power as compared to the latter. The evolution of 409 the average peak position and the average fwhm with incident 410 laser power densities for both bulk samples are shown in Figure 411 4, panels band c, respectively. The data displayed in Figure 4b,c 412 were averaged over measurements on four different spots on 413 each sample. The ${ }^{28} \mathrm{Si}_{0.6}{ }^{30} \mathrm{Si}_{0.4}$ and ${ }^{29} \mathrm{Si}$ bulk sample have peaks 414 at 514.9 and $512.3 \mathrm{~cm}^{-1}$, respectively. Unlike the case of $\mathrm{Si}$ 415 NWs, these phonon frequencies do not change significantly 416 with increasing laser power density. This is an expected 417 behavior because the effect of laser heating is ineffective in bulk 418 samples, which have higher thermal conductivities as compared 419 to NWs. The average fwhm, nearly $3 \mathrm{~cm}^{-1}$ smaller than those 420 measured for NWs, also shows a very limited increase with laser 421 power density that is almost identical for both bulk samples. It 422 is worth noting that the $\mathrm{Si}-\mathrm{Si}$ mode of the ${ }^{28} \mathrm{Si}_{0.6}{ }^{30} \mathrm{Si}_{0.4}$ bulk 423 sample is broader only by $0.4 \mathrm{~cm}^{-1}$ at the lowest incident laser 424 power than the $\mathrm{Si}-\mathrm{Si}$ mode of ${ }^{29} \mathrm{Si}$ bulk sample, which is 425 significantly less than the $3.2 \mathrm{~cm}^{-1}$ difference found between 426 the modes of the two sets of NWs. The difference of $0.4 \mathrm{~cm}^{-1}$ 427 between the average fwhm of the two bulk samples at the 428 lowest laser power density is not entirely coming from isotope 429 scattering effect. Indeed, the average mass of ${ }^{28} \mathrm{Si}_{0.6}{ }^{30} \mathrm{Si}_{0.4}$ being 430 smaller than the average mass of ${ }^{29} \mathrm{Si}$, the anharmonic scattering 431 of phonons, which scales inversely with average mass, is larger 432 in the ${ }^{28} \mathrm{Si}_{0.6}{ }^{30} \mathrm{Si}_{0.4}$ bulk sample as compared to that of the ${ }^{29} \mathrm{Si}$ 433 bulk sample at a fixed temperature. The contribution of this 434 excess anharmonic phonon scattering to the Raman line width 435 is hidden within the difference of $0.4 \mathrm{~cm}^{-1}$ between the average $436 \mathrm{fwhm}$ of the two bulk samples.

437 A plausible explanation of the observed broadening is the 438 nonuniform mixing of ${ }^{28} \mathrm{Si}$ and ${ }^{30} \mathrm{Si}$ isotopes during the VLS 439 growth of ${ }^{28} \mathrm{Si}_{x}{ }^{30} \mathrm{Si}_{1-x}$ NWs. Indeed, the excess broadening of 440 the Raman spectra for the ${ }^{28} \mathrm{Si}_{x}{ }^{30} \mathrm{Si}_{1-x}$ NWs probably originates 441 from the overlap of several narrower peaks corresponding to 442 different regions within a NW with different isotopic content. 443 For instance, in Figure 5a the Raman spectrum of a single ${ }_{444}{ }^{28} \mathrm{Si}_{x}{ }^{30} \mathrm{Si}_{1-x} \mathrm{NW}$ recorded at the lowest laser power is
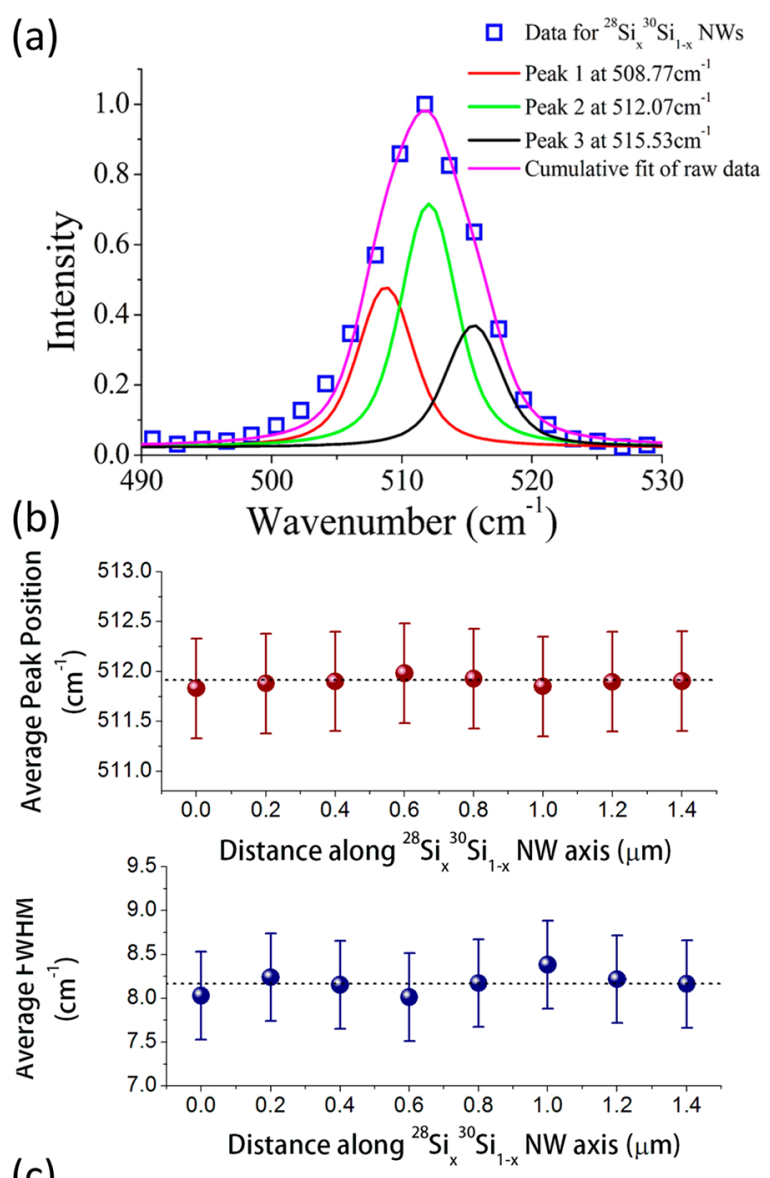

(c)

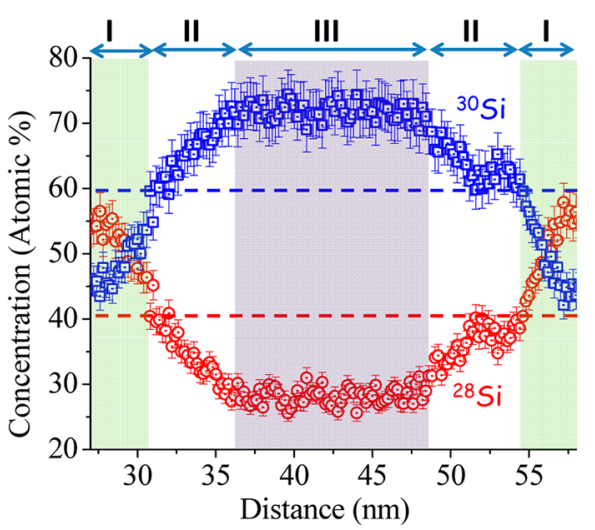

Figure 5. (a) The spectrum of a single ${ }^{28} \mathrm{Si}_{x}{ }^{30} \mathrm{Si}_{1-x} \mathrm{NW}$ at an incident power density of $0.08 \mathrm{~mW} / \mu \mathrm{m}^{2}$, data points shown in empty blue squares and the cumulative Voigt Fit (pink) has been simulated using the convolution of three different spectrum (red, green, and black) each representing different isotopic composition (details in text) within the NW; (b) peak position and fwhm profiles measured along the growth axis of single ${ }^{28} \mathrm{Si}_{x}{ }^{30} \mathrm{Si}_{1-x}$ nanowires. Each data point is an average over a few measurements on different nanowires. The horizontal dashed lines indicate the average values; (c) APT radial profile of ${ }^{28} \mathrm{Si}$ (red) and ${ }^{30} \mathrm{Si}$ (blue) isotopes across the diameter of an isotopically mixed nanowire. The offset in $x$-axis reflects the thickness of the $\mathrm{Ni}$ protective layer deposited around the nanowire to prevent any damage that may occur during FIB processing.

deconvoluted in three different peaks corresponding to a ${ }^{28} \mathrm{Si}-445$ rich area, a ${ }^{30} \mathrm{Si}$-rich area, and a transition zone. Because the 446 broadening due to isotopic scattering of phonons at $300 \mathrm{~K}$ is 447 only $0.065 \mathrm{~cm}^{-1}$ the fwhm of each of the three peaks has been 448 kept the same as that of an isotopically pure ${ }^{29} \mathrm{Si}$ NW. Peak 1449 
450 (red) is at $508.77 \mathrm{~cm}^{-1}$, peak 2 (green) is at $512.07 \mathrm{~cm}^{-1}$, and 451 peak 3 (black) is at $515.33 \mathrm{~cm}^{-1}$. The local compositions 452 corresponding to these three peaks are $26.9,45.8$, and $65.3 \%$, 453 respectively. The estimated uncertainty from the spectral 454 resolution of our Raman setup is about $7 \%$.

455 Interestingly, Raman spectra recorded along the growth axis 456 of individual ${ }^{28} \mathrm{Si}_{x}{ }^{30} \mathrm{Si}_{1-x}$ NWs show that neither the peak 457 position nor the fwhm of $\mathrm{Si}-\mathrm{Si}$ mode vary along the nanowire 458 growth axis (Figure $5 \mathrm{~b}$ ). This suggests that the isotopic content 459 is uniform along the growth axis and thus the inferred 460 nonuniformity of the isotopic content seems to be associated 461 with the radial distribution of the two isotopes. To verify this 462 intriguing observation, the nanowires investigated by Raman 463 were also analyzed using atom probe tomography (APT), 464 which is the only technique capable of providing the three465 dimensional (3D) distribution of different isotopes in a 466 nanoscale structure with a near atomic resolution. Details of 467 the APT analysis will be reported elsewhere. Figure 5c displays 468 the radial profiles of ${ }^{28} \mathrm{Si}$ and ${ }^{30} \mathrm{Si}$ isotopes across the diameter 469 of an isotopically mixed nanowire. The average isotopic 470 compostion as estimated from APT ${ }^{28} \mathrm{Si}_{0.41}{ }^{30} \mathrm{Si}_{0.59}$ which is 471 close to the content obtained from Raman analysis $472\left({ }^{28} \mathrm{Si}_{0.47}{ }^{30} \mathrm{Si}_{0.53}\right)$. Importantly, we note that, as predicted from 473 Raman spectra, APT analysis also confirms that the radial 474 distribution of the two isotopes is not uniform, whereas their 475 profiles along the growth axis (not shown here) remain 476 unchanged also in agreement with Raman data (Figure 5b). 477 Moreover, APT profiles demonstrate that the two isotopes are 478 distributed in three different regions (Figure 5c):

479 (1) Near the surface where ${ }^{28} \mathrm{Si}\left({ }^{30} \mathrm{Si}\right)$ content is higher 480 (lower) than its average content in the entire nanowire (region $481 \mathrm{I}$ ). The width of this region is about $26.3 \%$ of the nanowire 482 diameter.

483 (2) At the core of the nanowire where ${ }^{30} \mathrm{Si}\left({ }^{28} \mathrm{Si}\right)$ content is 484 higher (lower) than its average content in the entire nanowire 485 (region III). The width of this region is about $34.3 \%$ of the 486 nanowire diameter.

487 (3) A transition region between the two regions I and III 488 where the content of ${ }^{30} \mathrm{Si}\left({ }^{28} \mathrm{Si}\right)$ increases (decreases) 489 monotonically inward from nanowire surface to its core. The 490 width of this region is about $39.4 \%$ of the nanowire diameter. 491 The average isotopic composition of each region is $x(\mathrm{I})=$ $4920.50 \pm 0.01\left({ }^{28} \mathrm{Si}_{0.5}{ }^{30} \mathrm{Si}_{0.5}\right) ; x(\mathrm{II})=0.35 \pm 0.01\left({ }^{28} \mathrm{Si}_{0.35}{ }^{30} \mathrm{Si}_{0.65}\right)$; 493 and $x(\mathrm{III})=0.75 \pm 0.01\left({ }^{28} \mathrm{Si}_{0.25}{ }^{30} \mathrm{Si}_{0.75}\right)$. Clearly, APT analysis 494 confirms Raman-based observations reported above. At the 495 same time, the 3D atom-by-atom distribution of each isotope 496 within a single nanowire also raises fundamental questions 497 about the basic mechanisms and dynamics of the VLS growth. 498 Addressing these very important questions extends beyond the 499 main focus of this Letter.

500 In summary, we have demonstrated the growth of isotopi501 cally mixed Si NWs via the VLS process using isotopically 502 enriched silane precursors ${ }^{28} \mathrm{SiH}_{4},{ }^{29} \mathrm{SiH}_{4}$, and ${ }^{30} \mathrm{SiH}_{4}$. Using 503 Raman spectroscopy, the vibrational properties of these NWs 504 were investigated and compared to that of isotopically pure ${ }^{29} \mathrm{Si}$ 505 NWs having a close reduced mass. The outcome of the 506 comparative study indicates that there is an enhanced phonon 507 scattering in isotopically mixed NWs, which manifests itself at 508 two interrelated levels. First, the measured Raman spectra of 509 the ${ }^{28} \mathrm{Si}_{x}{ }^{30} \mathrm{Si}_{1-x}$ NWs were found to react to laser power quite 510 differently from those of ${ }^{29} \mathrm{Si}$ NWs. The redshift in peak 511 position and broadening of Raman spectra are more significant 512 for the former as compared to the latter with the local temperature of the ${ }^{28} \mathrm{Si}_{x}{ }^{30} \mathrm{Si}_{1-x}$ NWs at the highest power 513 density being almost $120 \mathrm{~K}$ above that of the ${ }^{29} \mathrm{Si}$ NWs. On the 514 basis of Raman nanothermometery, we estimated $230 \% 515$ reduction in the thermal conductivity of the ${ }^{28} \mathrm{Si}_{x}{ }^{30} \mathrm{Si}_{1-x} \mathrm{NWs} 516$ as compared to that of the ${ }^{29} \mathrm{Si} \mathrm{NWs}$ around $300 \mathrm{~K}$. Second, the 517 fwhm of the ${ }^{28} \mathrm{Si}_{x}{ }^{30} \mathrm{Si}_{1-x}$ NWs was found to be significantly 518 larger than that of ${ }^{29} \mathrm{Si}$ NWs regardless of the laser power. We 519 showed that this cannot come entirely from the isotope effect 520 and that the origin of this excess broadening might lie in 521 nonuniformity in mixing of the two isotope atoms within a 522 ${ }^{28} \mathrm{Si}_{x}{ }^{30} \mathrm{Si}_{1-x}$ NW. This nonuniform mixing of ${ }^{28} \mathrm{Si}$ and ${ }^{30} \mathrm{Si}$ may 523 unravel new insights into the dynamics of the VLS growth, 524 which extends beyond the scope of the current work. The work 525 presented here provides an essential body of information for 526 devices looking to exploit the thermal properties of NWs. The 527 results clearly show that the isotopically disordered and the 528 isotopically pure NWs respond to laser heating almost similarly 529 at low power ranges but their behavior differ drastically at high 530 power range. For NW-based devices, the isotopically mixed 531 NWs can be exploited for applications requiring lower thermal 532 conductivity, whereas the isotopically pure NWs are ideal for a 533 more efficient dissipation of heat.

\section{ASSOCIATED CONTENT}

534

\section{S Supporting Information}

535

Additional information including experimental details, addi- 537 tional figures, and additional references. The Supporting 538 Information is available free of charge on the ACS Publications 539 website at DOI: 10.1021/acs.nanolett.5b00708.

540

\section{AUTHOR INFORMATION}

\section{Corresponding Author}

*E-mail: oussama.moutanabbir@polymtl.ca.

Notes

The authors declare no competing financial interest.

\section{ACKNOWLEDGMENTS}

O.M. acknowledges funding from NSERC-Canada (Discovery 547 Grants) and Canada Research Chair, Fondation de l'École 548 Polytechnique de Montréal. The work at Keio was supported in 549 part by the Grant-in-Aid for Scientific Research by MEXT, in 550 part by NanoQuine, in part by FIRST, and in part by JSPS 551 Core-to-Core Program. J.A. acknowledges the funding from the 552 Generalitat de Catalunya 2014 SGR 1638. M.d.l.M. thanks the 553 CSIC Jae-Predoc program. J.A. and M.d.I.M. thank funding 554 from Spanish MINECO MAT2014-51480-ERC. The micros- 555 copy works have been conducted in the "Laboratorio de 556 Microscopias Avanzadas" at "Instituto de Nanociencia de 557 Aragon - Universidad de Zaragoza”. Authors acknowledge the 558 LMA-INA for offering access to their instruments and expertise, 559 especially to Dr. Cesar Magen.

\section{REFERENCES}

(1) Cardona, M.; Thewalt, M. L. W. Rev. Moden Phys. 2005, 77, 562 $1173-1224$

(2) Haller, E. E. MRS Bull. 2006, 31, 547-553.

(3) Hu, M.; Sinn, H.; Alatas, A.; Sturhahn, W.; Alp, E.; Wille, H.; 565 Shvyd'ko, Y.; Sutter, J.; Bandaru, J.; Haller, E.; Ozhogin, V.; Rodriguez, 566 S.; Colella, R.; Kartheuser, E.; Villeret, M. Phys. Rev. B 2003, 67, 567 113306.

(4) Davies, G.; Lightowlers, E. C.; Itoh, K.; Hansen, W. L.; Haller, E. 569 E.; Ozhogin, V. Semicond. Sci. Technol. 1992, 7, 1271-1273. 
571 (5) Tyryshkin, A. M.; Tojo, S.; Morton, J. J.; Riemann, H.; 572 Abrosimov, N. V.; Becker, P.; Pohl, H. J.; Schenkel, T.; Thewalt, M. L.; 573 Itoh, K. M.; Lyon, S. A. Nat. Mater. 2012, 11, 143-147.

574 (6) McCamey, D. R.; Van Tol, J.; Morley, G. W.; Boehme, C. Science 575 2010, 330, 1652-1656.

576 (7) Simmons, S.; Brown, R. M.; Riemann, H.; Abrosimov, N. V.; 577 Becker, P.; Pohl, H.-J.; Thewalt, M. L. W.; Itoh, K. M.; Morton, J. J. L. 578 Nature 2011, 470, 69-72.

579 (8) Itoh, K. M. Solid State Commun. 2005, 133, 747-752.

580 (9) Ozhogin, V. I.; Inyushkin, A. V.; Taldenkov, A. N.; Tikhomirov, 581 A. V.; Popov, G. É.; Haller, E.; Itoh, K. J. Exp. Theor. Phys. Lett. 1996, $58263,490-494$.

583 (10) Kremer, R. K.; Graf, K.; Cardona, M.; Devyatykh, G. G.; Gusev, 584 A. V.; Gibin, A. M.; Inyushkin, A. V.; Taldenkov, A. N.; Pohl, H. J. 585 Solid State Commun. 2004, 131, 499-503.

586 (11) Bracht, H.; Eon, S.; Frieling, R.; Plech, A.; Issenmann, D.; Wolf, 587 D.; Lundsgaard Hansen, J.; Nylandsted Larsen, A.; Ager, J. W., III; 588 Haller, E. E. New J. Phys. 2014, 16, 015021.

589 (12) Nakajima, M.; Harima, H.; Morita, K.; Itoh, K.; Mizoguchi, K.; 590 Haller, E. Phys. Rev. B 2001, 63, 161304.

591 (13) Morelli, D.; Heremans, J.; Slack, G. Phys. Rev. B 2002, 66, 592195304.

593 (14) Yang, N.; Zhang, G.; Li, B. Nano Lett. 2008, 8, 276-280.

594 (15) Hattori, J.; Uno, S. Jpn. J. Appl. Phys. 2013, 52, 04CN04.

595 (16) Moutanabbir, O.; Senz, S.; Zhang, Z.; Gösele, U. Nano Today 596 2009, 4, 393-398.

597 (17) Hannon, J. B.; Kodambaka, S.; Ross, F. M.; Tromp, R. M. 598 Nature 2006, 440, 69-71.

599 (18) Bailly, A.; Barrett, N.; Zagonel, L. F.; Gentile, P.; Pauc, N.; 600 Dhalluin, F.; Baron, T.; Chabli, A.; Cezar, J. C.; Brookes, N. B.; R, O. 601 Nano Lett. 2008, 8, 3709-3714.

602 (19) Adu, K.; Gutiérrez, H.; Kim, U.; Eklund, P. Phys. Rev. B 2006, $60373,155333$.

604 (20) Moutanabbir, O.; Miyamoto, S.; Haller, E. E.; Itoh, K. M. Phys. 605 Rev. Lett. 2010, 105, 026101.

606 (21) Balkanski, M.; Wallis, R.; Haro, E. Phys. Rev. B 1983, 28, 19286071934 .

608 (22) Scheel, H.; Reich, S.; Ferrari, A. C.; Cantoro, M.; Colli, A.; 609 Thomsen, C. Appl. Phys. Lett. 2006, 88, 233114.

610 (23) Soini, M.; Zardo, I.; Uccelli, E.; Funk, S.; Koblmüller, G.; 611 Fontcuberta i Morral, A.; Abstreiter, G. Appl. Phys. Lett. 2010, 97, 612263107.

613 (24) Frieling, R.; Radek, M.; Eon, S.; Bracht, H.; Wolf, D. E. Appl. 614 Phys. Lett. 2014, 105, 132104.

615 (25) Chen, S.; Wu, Q.; Mishra, C.; Kang, J.; Zhang, H.; Cho, K.; Cai, 616 W.; Balandin, A. A.; Ruoff, R. S. Nat. Mater. 2012, 11, 203-207.

617 (26) Widulle, F.; Ruf, T.; Konuma, M.; Silier, I.; Cardona, M.; 618 Kriegseis, W.; Ozhogin, V. I. Solid State Commun. 2001, 118, 1-22.

619 (27) Piscanec, S.; Ferrari, A. C.; Cantoro, M.; Hofmann, S.; Zapien, J. 620 A.; Lifshitz, Y.; Lee, S. T.; Robertson, J. Mater. Sci. Eng., C 2003, 23, 621 931-934.

622 (28) Fuchs, H. D.; Grein, C. H.; Cardona, M.; Hansen, W. L.; Itoh, 623 K.; Haller, E. E. Solid State Commun. 1992, 82, 225-228.

624 (29) Kittel, C. Introduction to Solid State Physics, 8th ed.; Wiley: New 625 York, 2004.

626 (30) Callaway, J. Phys. Rev. Lett. 1959, 113, 1046-1051.

627 (31) Gupta, R.; Xiong, Q.; Adu, C. K.; Kim, U. J.; Eklund, P. C. Nano 628 Lett. 2003, 3, 627-631.

629 (32) From ref $26, \Delta_{\text {Anhrm }}^{\text {Nat-Si Bulk }}(T=6 \mathrm{~K}) \approx 1.21 \mathrm{~cm}^{-1}$ and $\Delta_{\text {Isotope }}^{\text {Nat-Si Bulk }}$ 630 is negligible. This makes the anharmonic contribution equal to the 631 total line broadening from bulk ${ }^{\mathrm{Nat}} \mathrm{Si}$ sample. The anharmonic 632 contribution was extrapolated to $300 \mathrm{~K}$ using eq 3 , giving $\Delta_{\text {Anhrm }}^{\text {NatSi Bulk }}$ $633\left(T=300 \mathrm{~K} \approx \mathrm{fwhm}^{\mathrm{Nat}-\mathrm{Si}}(T=300 \mathrm{~K}) \approx 2.154 \mathrm{~cm}^{-1}\right.$. Next, a ${ }^{\text {Ant Si bulk }}$ 634 sample was measured using the InVia RM 3000 setup using $488 \mathrm{~nm}$ at 635 an incident power density of $0.08 \mathrm{~mW} / \mu \mathrm{m}^{2}$ and the fwhm was found 636 to be $\sim 5 \mathrm{~cm}^{-1}$. We attributed this excess line broadening $\left(\sim 3 \mathrm{~cm}^{-1}\right)$ of 637 InVia RM 3000 setup to instrumental resolution. We did not calculate 638 the contribution of instrumental broadening for the other setup (used 639 for high power measurements) because the entire analysis concerning the fwhm of the NWs was done using the data at the lowest recorded 640 power density which was recorded using the InVia RM 3000 setup. 641 (33) Moutanabbir, O.; Isheim, D.; Seidman, D. N.; Kawamura, Y.; 642 Itoh, K. M. Appl. Phys. Lett. 2011, 98, 013111. 